\title{
Efficient Graft Copolymerization of 2-Methyl-2-oxazoline onto Tosyl- and Iodo-Chitins in Solution
}

\author{
Keisuke Kurita, ${ }^{\dagger}$ Soichiro Hashimoto, Shigeru Ishil, \\ Tomonori MorI, and Shin-ichiro NishIMURA* \\ Department of Industrial Chemistry, Faculty of Engineering, Seikei University, \\ Musashino-shi, Tokyo 180, Japan \\ * Division of Biological Science, Graduate School of Science, Hokkaido University, \\ Sapporo 060, Japan
}

(Received January 12, 1996)

\begin{abstract}
Graft copolymerization of 2-methyl-2-oxazoline onto tosyl- and iodo-chitins has been examined. The reaction proceeded efficiently with tosyl-chitin in solution to give chitin derivatives having poly ( $N$-acetylethyleneimine) side chains, and the grafting percentage was $280 \%$ under appropriate conditions. Graft copolymerization was also possible with iodo-chitin, but rather sluggish. Hydrolytic degradation of the chitin main chain allowed the isolation of the introduced side chains. The number-average molecular weight of the polyethyleneimine isolated from a graft copolymer with grafting percentage of $160 \%$ was 2700 , indicating that $18 \%$ of the carbon atoms having a tosyloxy group were actually utilized for initiating the graft copolymerization. The resulting graft copolymers showed high affinity for both organic solvents and water, and may be useful as a new type of chitin-derived hybrid materials.

KEY WORDS Chitin / Chemical Modifications / Tosyl-Chitin / Iodo-Chitin / Oxazoline / Graft Copolymerization / Grafting Percentage / Poly $(N$-acetylethyleneimine $) /$ Polyethyleneimine / Solubility
\end{abstract}

Although chitin is an attractive polysaccharide produced in a huge amount annually in nature, it has remained an almost unutilized biomass resource. Chitin is structurally close to cellulose, only the substituent at $\mathrm{C}-2$ being different. It is an amino polysaccharide having acetamide groups at C-2 and thus expected to have higher potential as a specialty polymeric material than cellulose bearing only hydroxyl groups.

Chemical modifications will open ways to various utilizations of this abundant natural polymer, but they generally encounter difficulty owing to the inherent intractable nature of chitin. Of possible modifications, graft copolymerization is anticipated to be quite promising for developing sophisticated functions; it would enable a wide variety of molecular designs to afford novel types of tailored hybrid materials composed of natural polysaccharides and synthetic polymers. The properties of the resulting graft copolymers may be widely controlled by the characteristics of the side chains including molecular structure, length, and number. Moreover, chitin-based graft copolymers are interesting in view of further modifications possible with the versatile $\mathrm{C}-2$ acetamide groups.

Recently, acrylamide, ${ }^{1}$ acrylic acid, ${ }^{1}$ methyl acrylate, ${ }^{2}$ and methyl methacrylate ${ }^{3}$ were graft-copolymerized onto chitin in water with cerium(IV). Graft copolymerization is also possible with tributylborane ${ }^{4}$ and $\gamma$-ray ${ }^{5}$ or UV irradiation. ${ }^{6}$ However, the reactions have to be conducted under heterogeneous conditions in suspension owing to the insolubility of chitin and are thus generally poor in efficiency and reproducibility. Though insoluble, mercapto-chitin is a suitable radical initiator for styrene ${ }^{7}$ and methyl methacrylate ${ }^{8}$ to introduce side chains at the labile mercapto groups. As a new approach to graft copolymers, living poly(2-oxazoline)s were terminated

† To whom all correspondence should be addressed. with the amino groups of partially deacetylated chitin to give chitin derivatives having poly(2-oxazoline) chains. ${ }^{9}$

Soluble chitin derivatives are expected to make possible the efficient and controlled graft copolymerization and will solve the problems associated with the insolubility of chitin. The water-soluble chitin, ${ }^{10}$ a derivative having acetyl groups at about half of the amino groups randomly, allows efficient graft copolymerization of $\alpha$-amino acid $N$-carboxy anhydrides in aqueous solution. ${ }^{11-13}$ Iodo-chitin, soluble in organic solvents, has proved an appropriate precursor for graft copolymerization of styrene by either a cationic or radical mechanism. ${ }^{14}$

Tosyl-chitin, ${ }^{15,16}$ as well as iodo-chitin, is another promising organo-soluble precursor. Because of the solubility and presence of carbon atoms with considerable cationic character, tosyl- and iodo-chitins would be suitable initiators for the efficient cationic ring-opening polymerization of oxazolines in solution. As suggested by the improved solubility of the graft copolymers prepared from tosyl-cellulose and 2-oxazolines, ${ }^{17}$ the introduction of poly(2-oxazoline) side chains into chitin will serve to impart solubility. Tosyl-chitin shows considerably higher solubility than tosyl-cellulose as a result of the homogeneous preparation conditions and hence probable random distribution of tosyl groups, ${ }^{16}$ and thus the derived graft copolymers would exhibit high affinity for both organic solvents and water. This paper describes the graft copolymerization behavior of 2 methyl-2-oxazoline onto tosyl- and iodo-chitins in solution and some properties of the resulting copolymers.

\section{EXPERIMENTAL}

\section{General}

2-Methyl-2-oxazoline was purified by distillation. $N, N$-Dimethylformamide (DMF), $N, N$-dimethylacet- 
amide (DMAc), and dimethyl sulfoxide (DMSO) were dried with calcium hydride, distilled in nitrogen under reduced pressure, and stored over $3 \AA$ molecular sieves. IR spectra were recorded with a JASCO IR-700. Conductometric titration was carried out in aqueous DMSO with a conductivity meter TOA CM-40S to determined the amounts of free amino groups. ${ }^{16} \mathrm{GPC}$ was performe with a JASCO 880-PU connected to a Shodex RI detector SE-61 (column, Shodex KD-806M + KD802; solvent, DMF; standards, polystyrene).

\section{Tosyl- and Iodo-Chitins}

Tosylation of chitin was carried out according to the interfacial procedure reported recently, ${ }^{16}$ and the tosylchitin used in this study had a degree of substitution (ds) of 0.67 or 0.78 . Iodo-chitin was prepared from a tosylchitin with ds 0.67 by treating with sodium iodide in DMSO solution. ${ }^{16}$

\section{Graft Copolymerization}

Tosyl-chitin (ds $0.67,0.80 \mathrm{~g}$ ) was dispersed in $60 \mathrm{~mL}$ of DMAc, and the mixture was stirred at room temperature for $3 \mathrm{~h}$ to give an almost homogeneous solution. To the mixture was added $44.0 \mathrm{~g}$ ( 200 equiv to pyranose; 300 equiv to tosyl) of 2-methyl-2-oxazoline. The reaction was carried out with stirring in a nitrogen atmosphere at $80^{\circ} \mathrm{C}$ for $48 \mathrm{~h}$, and the resulting light yellow solution was poured into ether to give a white precipitate. It was collected by centrifugation and washed with dichloromethane/acetone $(9: 1)$ overnight and then with ether overnight. The precipitate was collected by centrifugation and dried to give $0.52 \mathrm{~g}$ of the graft copolymer (chitin-graft-poly ( $N$-acetylethyleneimine)) as a white solid. IR (KBr): $v 3440(\mathrm{OH}$ and $\mathrm{NH}), 1635$ $(\mathrm{C}=\mathrm{O})$, and $1100-1000 \mathrm{~cm}^{-1}$ (pyranose).

The combined supernatants and washings were concentrated under reduced pressure and poured into ether to give a white precipitate. It was collected by centrifugation and dried to give $3.10 \mathrm{~g}$ of the homopolymer of 2-methyl-2-oxazoline, poly( $N$-acetylethyleneimine).

\section{Isolation of the Side Chains}

A dispersion of a chitin-graft-poly $(N$-acetylethyleneimine) (grafting, $160 \% ; 0.40 \mathrm{~g}$ ) in $300 \mathrm{~mL}$ of $12 \mathrm{~mol} \mathrm{~L}^{-1}$ hydrochloric acid was heated at $100^{\circ} \mathrm{C}$ for $24 \mathrm{~h}$ with stirring. The resulting brown solution was cooled to room temperature and neutralized with $12 \mathrm{~mol} \mathrm{~L}^{-1}$ sodium hydroxide. Water was evaporated under reduced pressure to give a light brown material. It was extracted with $400 \mathrm{~mL}$ of chloroform, and the extract was concentrated under reduced pressure to give $0.073 \mathrm{~g}$ ( $30 \%$ yield) of polyethyleneimine as a light brown semi-solid. The number-average molecular weight, $M_{n}$, was 2700 as determined by GPC in DMF.

\section{RESULTS AND DISCUSSION}

Polymerization of 2-oxazolines can be initiated cationically with alkyl halides, fluorinated Lewis acids, sulfonate and sulfate esters, or mineral acids to give poly $(N$-acylethyleneimine $)$ s. ${ }^{18}$ Tosyl- and iodo-chitins are soluble in solvents unlike most other chitin derivatives and thus should be appropriate initiators for the polymerization of 2-methyl-2-oxazoline to afford the graft copolymers, chitins having poly $(N$-acetylethyleneimine) side chains.

\section{Tosyl- and Iodo-Chitins}

Tosylation of chitin was effected by the interfacial method between an alkali chitin solution and a chloroform solution of tosyl chloride. The reaction is quite efficient at low temperatures, but $N$-deacetylation takes place to some extent because of the alkaline conditions. Since the free amino groups may interfere with the graft copolymerization and also make difficult structural identification of the products, they were acetylated with acetic anhydride in methanol. Hydroxyl groups were acetylated at the same time, though to only a limited extent, and thus $O$-deacetylation was subsequently conducted with potassium hydroxide in methanol. ${ }^{16}$ Conductometric titration of the products confirmed the absence of the free amino groups. The resulting tosyl-chitin with a ds of 0.67 or 0.78 was soluble in aprotic polar solvents such as DMF, DMAc, and DMSO.

On treatment of the tosyl-chitin dissolved in DMSO with sodium iodide, substitution reaction proceeded in solution, giving rise to the corresponding iodo-chitin. ${ }^{16}$ It showed even better solubility in organic solvents than tosyl-chitin.

\section{Graft Copolymerization}

Dissolution of tosyl-chitin in DMF, DMAc, or DMSO sometimes takes several hours at room temperature, and thus the monomer, 2-methyl-2-oxazoline, was added after an almost homogeneous or highly swollen mixture resulted. The reaction is initiated by a cationic mechanism, and the ring-opening polymerization of 2-methyl-2-oxazoline occurred at $80^{\circ} \mathrm{C}$ according to Scheme 1. As the polymerization proceeded, the mixture became a clear solution. The resulting graft copolymer was isolated as a white precipitate by pouring the solution into ether followed by washing thoroughly with a mixture of dichloromethane and acetone to remove the monomer and poly( $N$-acetylethyleneimine $)$ homopolymer.

Grafting behavior is discussed in terms of the grafting percentage which is a ratio of the weight of introduced side chains to that of the polymer main chain. The grafting percentage was determined with a calibration line based on IR spectroscopy with mixtures of chitin and $\operatorname{poly}(N$-acetylethyleneimine) homopolymer; the absorbance ratios of a band at $1419 \mathrm{~cm}^{-1}$ characteristic of poly $(N$-acetylethyleneimine $)$ to a band at $1070 \mathrm{~cm}^{-1}$ due to pyranose rings were plotted against the mixing

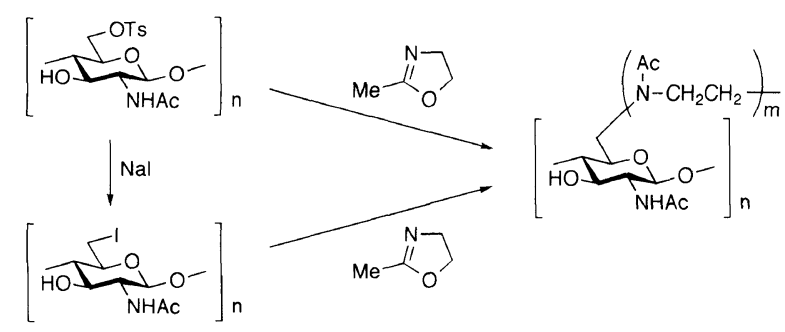

Scheme 1. 
Table 1. Graft copolymerization of 2-methyl-2-oxazoline onto tosyl- and iodo-chitins

\begin{tabular}{|c|c|c|c|c|c|}
\hline Chitin derivative & & Solvent & Temp & Yield & Grafting \\
\hline $\mathrm{mg}$ & pyranose $^{\mathrm{a}}$ & $\mathrm{mL}$ & ${ }^{\circ} \mathrm{C}^{\mathrm{b}}$ & $\mathrm{mg}$ & $\%^{\mathrm{c}}$ \\
\hline Tosyl-chitin ${ }^{\mathrm{d}}(410)$ & 200 & DMF (30) & 80 & 230 & 80 \\
\hline Tosyl-chitin ${ }^{\mathrm{d}}(800)$ & 200 & DMAc $(60)$ & 80 & 520 & 160 \\
\hline Tosyl-chitin ${ }^{\mathrm{e}}(100)$ & 300 & DMAc (6) & 80 & 320 & 280 \\
\hline Tosyl-chitin $^{\mathrm{e}}(100)$ & 400 & DMAc (6) & 80 & 90 & 220 \\
\hline Iodo-chitin ${ }^{\mathrm{f}}(50)$ & 65 & $\operatorname{DMF}(5)$ & 80 & 44 & 0 \\
\hline Iodo-chitin $^{\mathrm{f}}(50)$ & 65 & DMF (5) & 120 & 50 & 10 \\
\hline
\end{tabular}

${ }^{\mathrm{a}}$ Molar ratio. ${ }^{\mathrm{b}}$ Reaction time, $48 \mathrm{~h} .{ }^{\mathrm{c}}$ Grafting $(\%)=($ weight of branches/weight of main chains $) \times 100 .{ }^{\mathrm{d}} \mathrm{ds} 0.67 .{ }^{\mathrm{e}} \mathrm{ds} 0.78 .{ }^{\mathrm{f}}$ Prepared from tosyl-chitin with ds 0.67 .

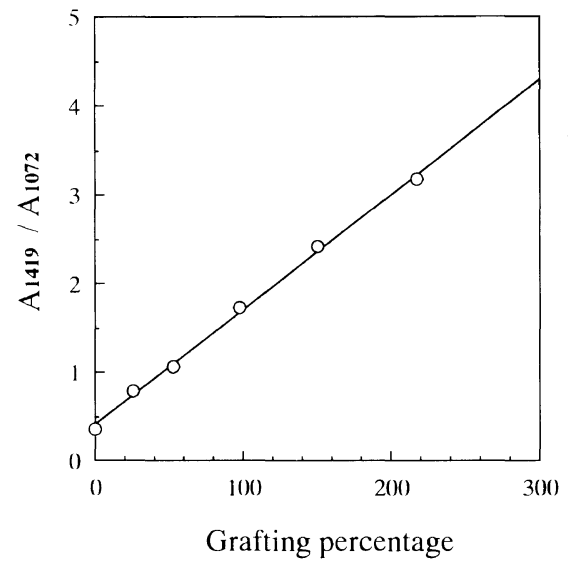

Figure 1. Calibration line to determine grafting percentages.

ratios, hence grafting percentages (Figure 1). As a polymerization solvent, DMAc appeared more suitable than DMF, judging from the grafting percentage and yield in Table I. The grafting percentage was dependent on the amount of 2-methyl-2-oxazoline and reached $280 \%$ under appropriate conditions.

Graft copolymerization was also attempted with iodo-chitin, but no grafting was observed under similar conditions. When the reaction temperature was raised to $120^{\circ} \mathrm{C}$, the oxazoline was graft-copolymerized though the grafting percentage was low. This indicates that the carbon bearing a tosyloxy group is superior to that having an iodo group with regard to the ability of initiating the cationic ring-opening polymerization of the oxazoline.

\section{Characterization of Graft Copolymers}

The IR spectra of the graft copolymers were similar to the spectrum of $\operatorname{poly}(N$-acetylethyleneimine) homopolymer when the grafting percentage was above $100 \%$ as illustrated in Figure 2. A strong band at $1635 \mathrm{~cm}^{-1}$ is attributable to the $N$-acetyl groups of both the chitin main chain and poly $(N$-acetylethyleneimine) branches. Bands due to the tosyl groups including a characteristic sharp one at $1176 \mathrm{~cm}^{-1}$ disappeared in the IR spectra of the graft copolymers, indicating the absence of tosyl groups after isolation.

The resulting graft copolymers showed extraordinary solubility unlike insoluble chitin. They were soluble in aprotic polar solvents such as DMSO and DMF when the grafting percentage was above $80 \%$ and swelled highly even in low-boiling common solvents. They also showed high affinity for water, but the solubility was sometimes dependent on the state of the solids after 688

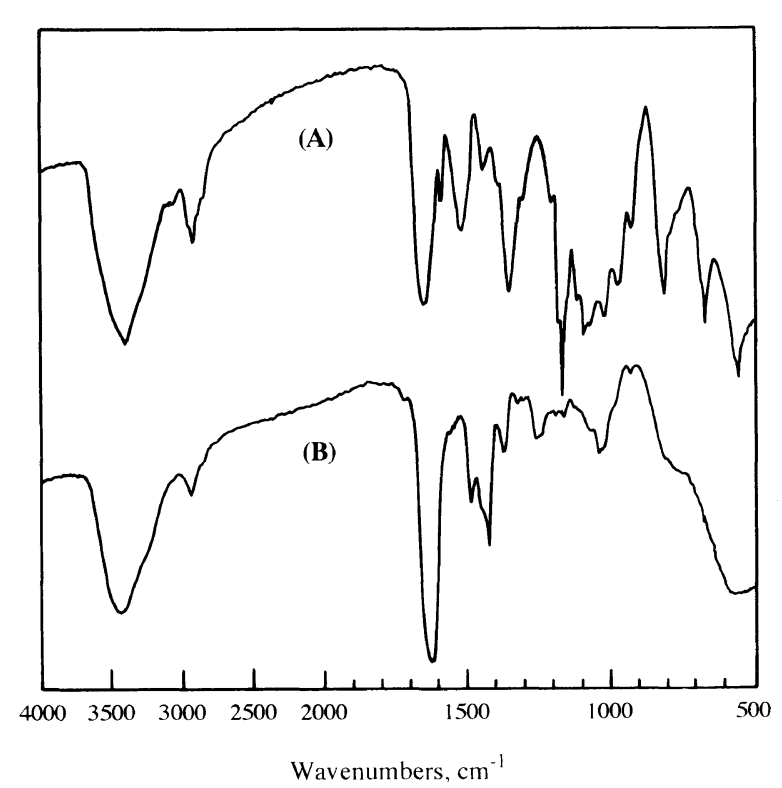

Figure 2. IR spectra of (A) tosyl-chitin with ds 0.67 and (B) graft copolymer with grafting percentage of $220 \%$ ( $\mathrm{KBr}$ method).

Table II. Solubility of graft copolymers ${ }^{\mathrm{a}}$

\begin{tabular}{cccccc}
\hline $\begin{array}{c}\text { Grafting/ } \\
\%\end{array}$ & DMSO & DMF & $\mathrm{CHCl}_{3}$ & $\mathrm{MeOH}$ & $\mathrm{H}_{2} \mathrm{O}$ \\
\hline 10 & \pm & \pm & \pm & \pm & \pm \\
80 & + & + & \pm & $+( \pm)^{\mathrm{b}}$ & $+\stackrel{( \pm)^{\mathrm{b}}}{ \pm}$ \\
160 & + & + & \pm & $+( \pm)^{\mathrm{b}}$ & $+( \pm)^{\mathrm{b}}$ \\
\hline
\end{tabular}

${ }^{\mathrm{a}}+$, soluble; \pm , partially soluble or swelled. ${ }^{\mathrm{b}}$ Solubility is dependent on the state of the copolymers after isolation, as discussed in the text.

drying as listed in Table II. They were soluble in water in some cases, in particular when they were isolated as fluffy materials, but not completely soluble when they were solid masses.

\section{Characterization of Side Chains}

To elucidate the length and number of the introduced side chains and also to discuss the initiating efficiency of the tosyl-chitin, the chitin backbone of the graft copolymer was hydrolyzed with hydrochloric acid (Scheme 2). Complete degradation was confirmed by dissolution into the hydrochloric acid, as in the hydrolysis of chitin ${ }^{19}$ and chitin-graft-polystyrene, ${ }^{7}$ and the liberated polyethyleneimine was subjected to GPC analysis. When the graft copolymer with grafting percentage of $160 \%$ prepared from the tosyl-chitin with ds 0.67 was degraded, the isolated polyethyleneimine had an $M_{n}$ val- 


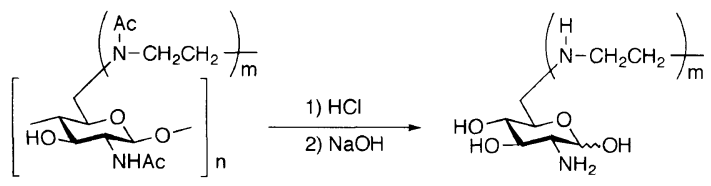

Scheme 2.

ue of 2700. On the basis of this value along with those of the ds of tosyl-chitin and grafting percentage, the ratio of tosylate groups actually utilized for initiating the graft copolymerization was calculated to be $18 \%$. This indicates that a side chain is attached on the average to every eight pyranose units. The high initiation efficiency achieved in this graft copolymerization is ascribable to the efficient grafting in homogeneous solution.

\section{CONCLUSION}

Both tosyl- and iodo-chitins can be used to initiate the graft copolymerization of 2-methyl-2-oxazoline by a cationic mechanism in homogeneous solution, but tosylchitin is a more suitable precursor for the reaction under mild conditions. Grafting behavior is interesting judging from the high initiation efficiency of the tosylate groups. The resulting hybrid materials may find various applications owing to the marked affinity for both organic solvents and water and the possible further modifications characteristic of the functionality of chitin.

\section{REFERENCES AND NOTES}

1. K. Kurita, M. Kawata, Y. Koyama, and S. Nishimura, J. Appl. Polym. Sci., 42, 2885 (1991).
2. A. Lagos, M. Yazdani-Pedram, J. Reyes, and N. Campos, J. Macromol. Sci., Pure Appl. Chem., A29, 1007 (1992).

3. L. Ren, Y. Miura, N. Nishi, and S. Tokura, Carbohydr. Polym., 21, 23 (1993).

4. K. Kojima, M. Yoshikuni, and T. Suzuki, J. Appl. Polym. Sci., 24, 1587 (1979).

5. Y. Shigeno, K. Kondo, and K. Takemoto, J. Macromol. Sci., Chem., A17, 571 (1982).

6. A. Takahashi, Y. Sugahara, and Y. Hirano, J. Polym. Sci., Part A: Polym. Chem., 27, 3817 (1989).

7. K. Kurita, S. Hashimoto, H. Yoshino, S. Ishii, and S. Nishimura, Macromolecules, 29, 1939 (1996).

8. K. Kurita, S. Hashimoto, S. Ishii, and T. Mori, Polym. Bull., in press.

9. K. Aoi, A. Takasu, and M. Okada, Macromol. Chem. Phys., 195, 3835 (1994).

10. K. Kurita, T. Sannan, and Y. Iwakura, Makromol. Chem., 178, 3197 (1977).

11. K. Kurita, M. Kanari, and Y. Koyama, Polym. Bull., 14, 511 (1985).

12. K. Kurita, A. Yoshida, and Y. Koyama, Macromolecules, 21, 1579 (1988).

13. K. Kurita, S. Iwawaki, S. Ishii, and S. Nishimura, J. Polym. Sci., Part A: Polym. Chem., 30, 685 (1992).

14. K. Kurita, S. Inoue, K. Yamamura, H. Yoshino, S. Ishii, and S. Nishimura, Macromolecules, 25, 3791 (1992).

15. K. Kurita, S. Inoue, and S. Nishimura, J. Polym. Sci., Part A: Polym. Chem., 29, 937 (1991).

16. K. Kurita, H. Yoshino, K. Yokota, M. Ando, S. Inoue, S. Ishii, and S. Nishimura, Macromolecules, 25, 3786 (1992).

17. S. Kobayashi, M. Kaku, and T. Saegusa, Macromolecules, 21, 1921 (1988)

18. For example; (a) A. Tomalia and D. P. Sheetz, J. Polym. Sci. A-1, 4, 2253 (1963); W. Seeliger, Angew. Chem., 78, 613, 913 (1966); T. Kagiya, S. Narisawa, T. Maeda, and K. Fukui, J. Polym. Sci., B, 4, 441 (1966); T. G. Bassiri, A. Levy, and M. Litt, J. Polym. Sci., B, 5, 871 (1967); (b) T. Saegusa, H. Ikeda, and H. Fuji, Polym. J., 3, 35 (1972).

19. E. R. Purchase and C. E. Braun, Org. Synth., Coll. Vol. 3, 430 (1955). 